\title{
Pautas ecocardiográficas de la vista de cuatro cámaras para el diagnóstico prenatal del retorno venoso pulmonar anómalo
}

Esteban Vázquez*

Alberto Sosa Olavarría**

Natalia Soria Nóbrega*

*Hospital Interzonal General de Agudos "San Marín" de La Plata. Buenos Aires. Argentina

** Universidad de Carabobo. Valencia. Venezuela

Recibido: 30/05/2020

Aceptado: 12/06/2020

En línea: 31/07/2020

\section{Correspondencia}

Esteban Vázquez

esteban.cardiologiainfantil@gmail.com

Citar como: Vázquez E, Sosa-Olavarría A, Soria-Nóbrega N. Pautas ecocardiográficas de la vista de cuatro cámaras para el diagnóstico prenatal del retorno venoso pulmonar anómalo. Rev Ecocar Pract (RETIC). 2020 (Jul); 3 (2): 55-57. doi: 10.37615/retic.v3n2a17.

Cite this as: Vázquez E, Sosa-Olavarría A, Soria-Nóbrega N. Echocardiographic guidelines of the 4-chamber view for the prenatal diagnosis of Abnormal Pulmonary Venous Return. Rev Ecocar Pract (RETIC). 2020 (Jul); 3 (2): 55-57. doi: 10.37615/retic.v3n2a17.

\section{Palabras clave}

$\triangleright$ Ecocardiografía fetal

$\triangleright$ Retorno venoso pulmonar anómalo

\section{Keywords}

$\triangleright$ Fetal ecocardiography

$\triangleright$ Abnormal pulmonary venous return

\section{RESUMEN}

Se presenta un resumen práctico de los hallazgos más frecuentes al hacer un diagnóstico prenatal de retorno venoso pulmonar anómalo. Se describe cómo es importante visualizar el área retrocardíaca de manera rutinaria, sobre todo mediante el plano de corte de cuatro cámaras. Conocer las características ecocardiográficas prenatales de esta anomalía es importante por la dificultad que presenta su diagnóstico.

\section{Retorno venoso pulmonar anómalo}

El retorno venoso pulmonar anómalo (RVPA) puede clasificarse como tota ${ }^{(1)} \mathrm{O}$ parcial. Se distinguen los siguientes tipos:

- Supracardíaco-l.

- Cardíaco-Il.

- Infradiafragmático-lll o mixto.

El área retrocardíaca debe ser interrogada de manera rutinaria.

La visualización de las VsPs ingresando a la pared posterior y lateral de la aurícula izquierda (Vídeo 1) se indican en numerosas guías de cribado y aun así el RVPA sigue siendo, quizá, la cardiopatía congénita con mayor subdiagnóstico.

Los signos ecocardiográficos presentados en el estudio del corte de cuatro cámaras pueden ayudar a aumentar la detección de esta cardiopatía:
1. Drenaje VsPs. La falta de visualización de las VsPs ingresando en la aurícula izquierda (Vídeo 2) otorga una forma "lisa" a la pared posterior de la aurícula (Figura 1).

2. Colector o vena confluente. La presencia de una estructura vascular puede detectarse en el área retrocardíaca en modo bidimensional, pero se recomienda el uso del Doppler-color. Cuando su trayecto es horizontal, se ha descrito como signo de "la ramita"(2) (Vídeo 3). En cambio el RVPA III mostrará el colector en un eje axial (Vídeo 4).

3. Índice del espacio posterior a la AI. "Post-LA space index"(3) es un marcador potencial de RVPA total aislado. El aumento de la distancia entre la pared anterior de la aorta torácica y la pared posterior de la Al en los casos de RVPA total (Figura 2).

4. Dilatación del seno coronario (SC). Este signo cobra importancia en ausencia de visualización de vena cava superior izquierda persistente. La detección de las venas pulmonares desembocando en el SC confirma RVPA tipo II.

5. Asimetría de cavidades cardíacas (ACC). No es un signo constante ni patognomónico. A medida que avanza la edad gestacional, el RVPA puede presentar dominancia de cavidades derechas (Figura 3). 


\section{Estudio por imagen}

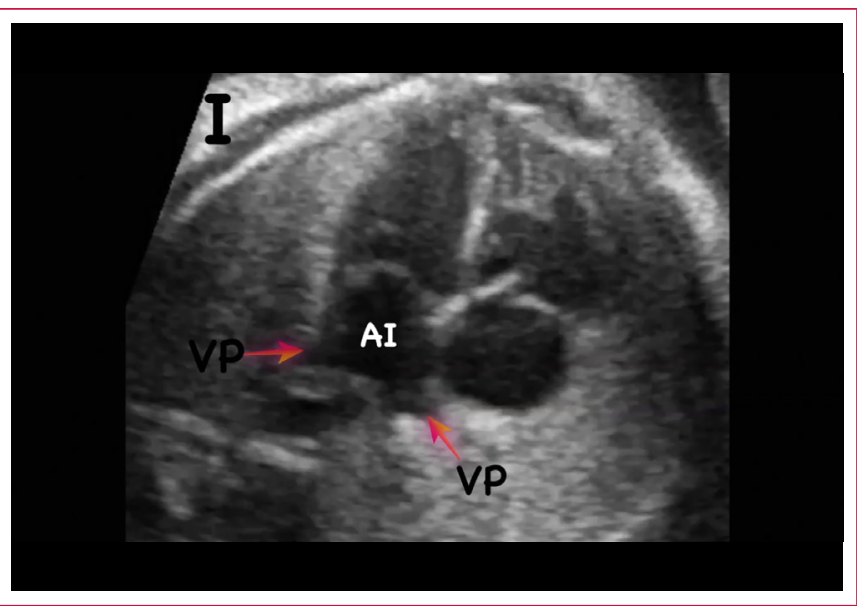

Vídeo 1. VsPs inferiores (Al: aurícula izquierda; flechas: VsPs inferiores)

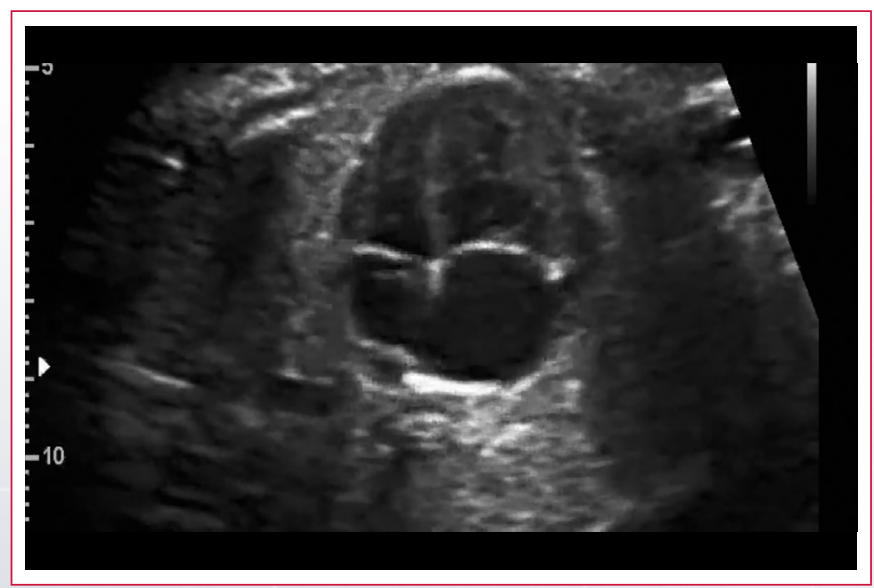

Vídeo 2. RVPA total tipo III. Ausencia del ingreso de las VsPs inferiores a la Al

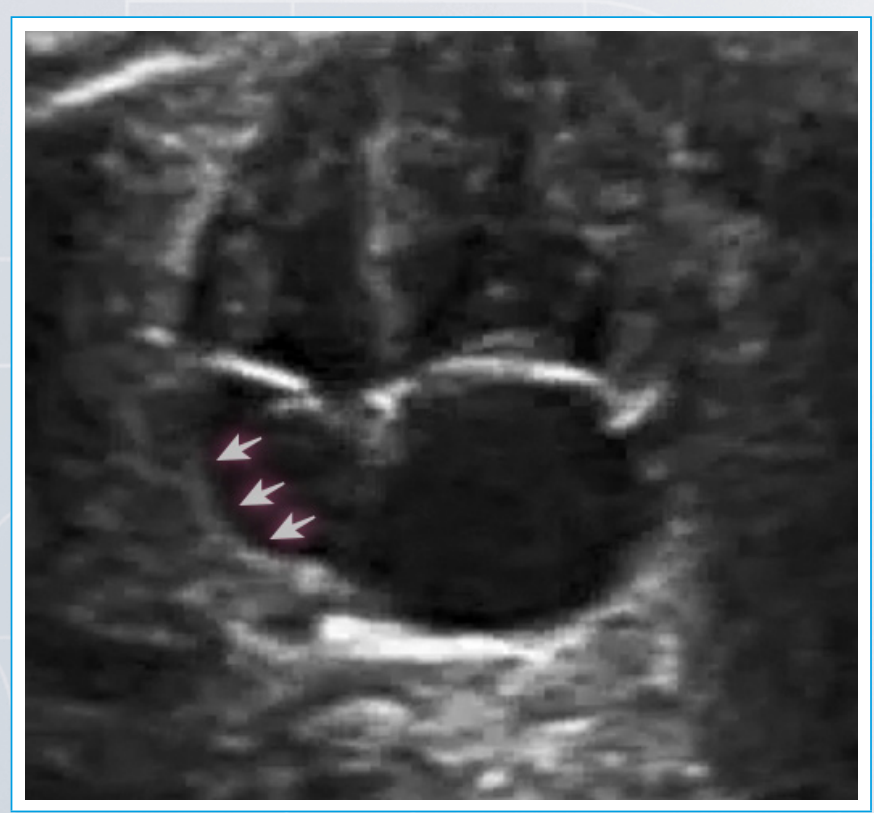

Figura 1. RVPA total tipo III. Flechas: ausencia de venas pulmonares ingresando a la Al y forma lisa de la pared posterior de la cámara

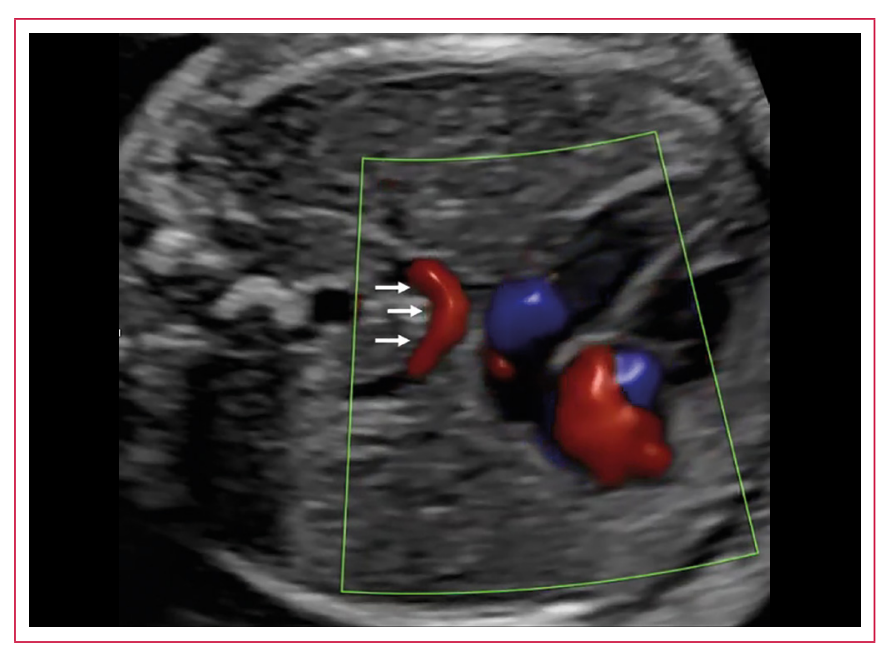

Vídeo 3. RVPA total tipo I supracardíaca. Flechas: colector. Signo de "la ramita"

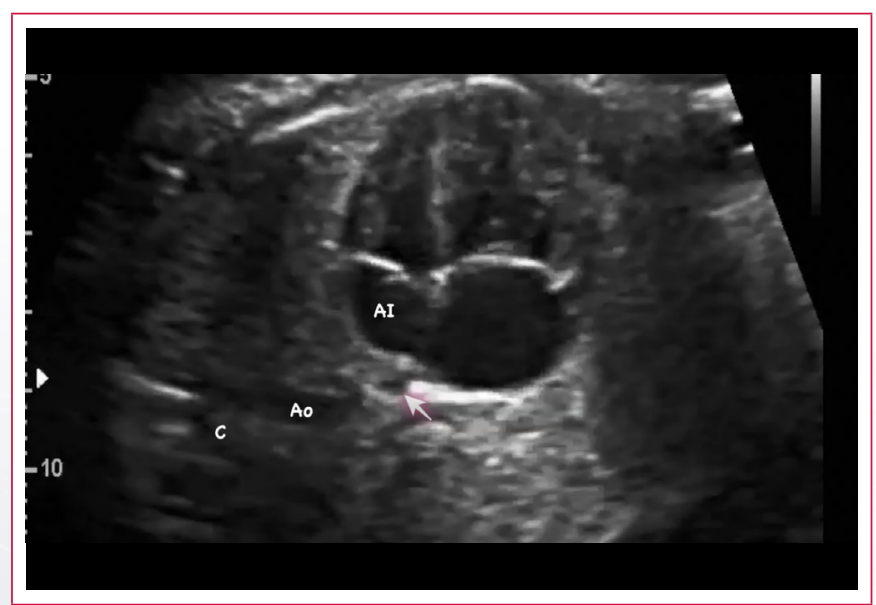

Vídeo 4. RVPA total tipo III. Flecha: colector

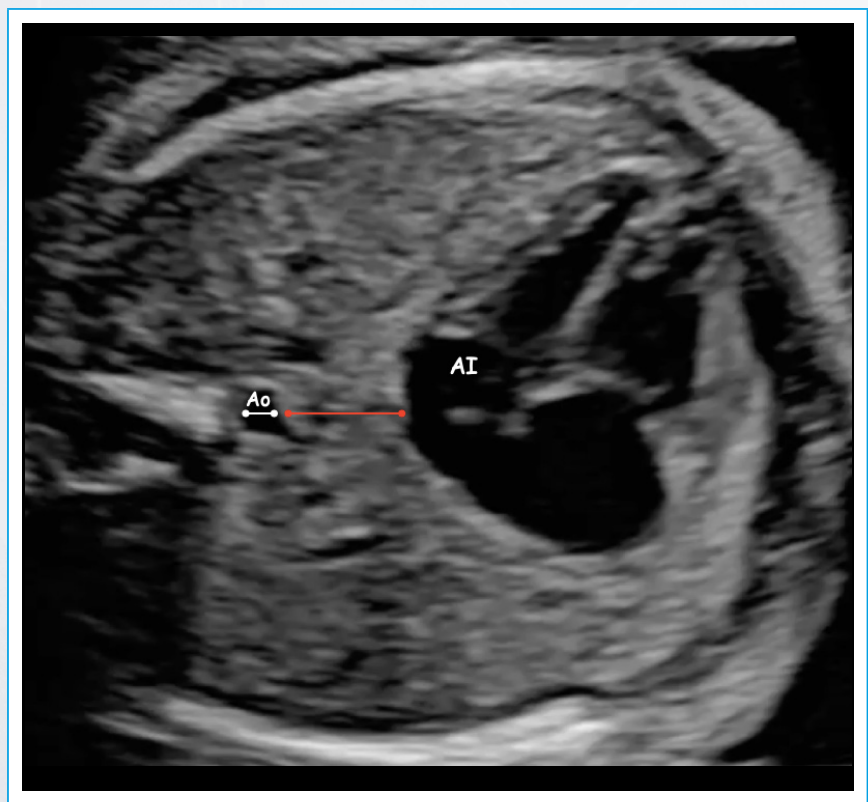

Figura 2. RVPA total tipo I. La distancia entre la cara anterior de la aorta torácica y la pared posterior de la Al (rojo) es mayor al diámetro de la aorta torácica (blanca) 


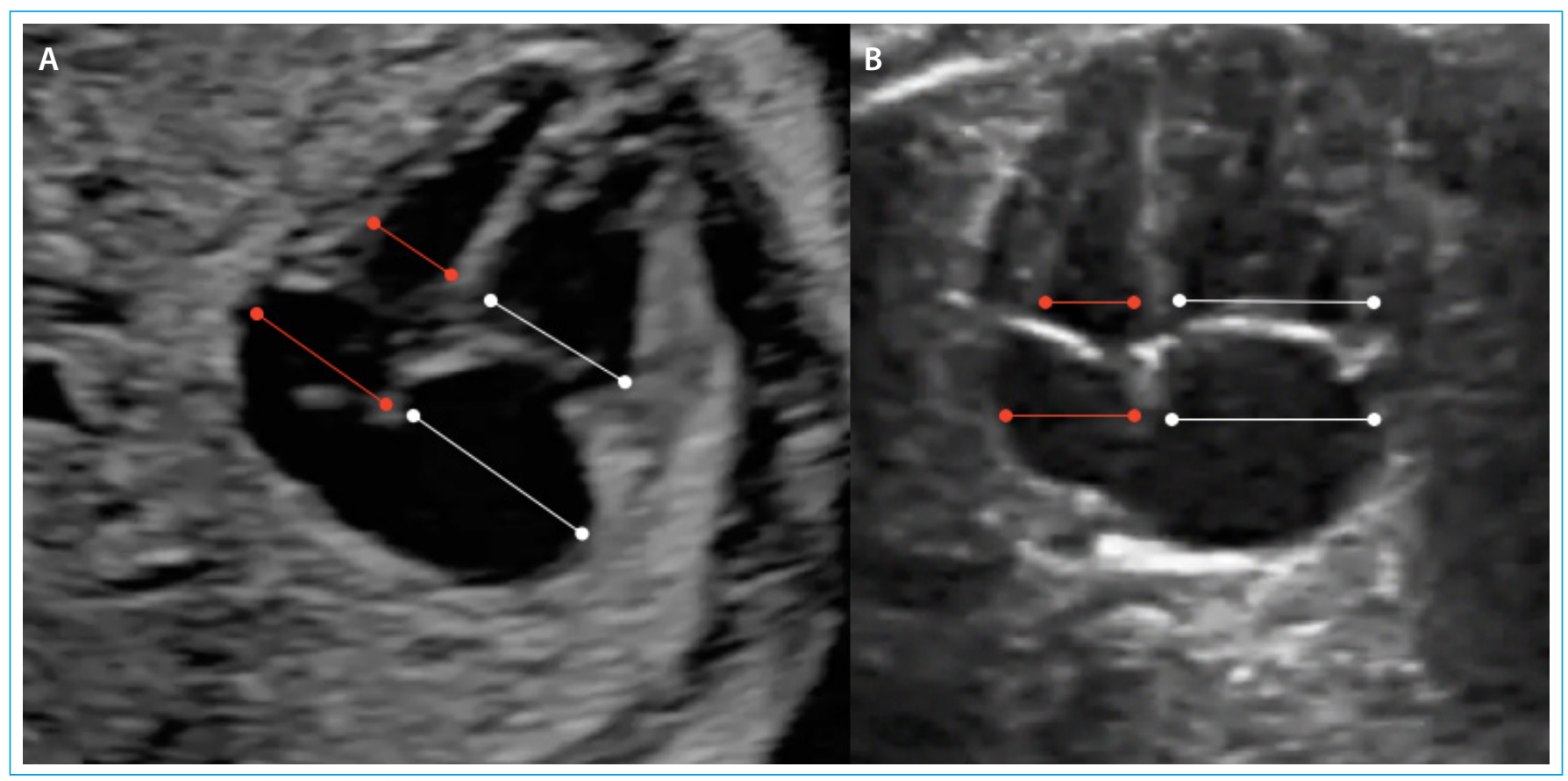

Figura 3. ACC con dominancia derecha. A: RVPA total tipo I. B: RVPA total tipo III. Líneas rojas: cavidades izquierdas; líneas blancas: cavidades derechas

\section{Ideas para recordar}

- La sospecha y el diagnóstico del RVPA continúa siendo un desafío permanente para el operador, con las tasas más bajas de detección prenatal.

- Se presentan hallazgos del cribado de cardiopatías congénitas compatibles con dicha patología: drenaje VsPs, colector o vena confluente, índice del espacio posterior a la Al, dilatación del seno coronario y asimetría de cavidades cardíacas.

\section{Bibliografía}

1. Seale A, Carvalho JS, Gardiner HM, et al. Total anomalous pulmonary venous connection: impact of prenatal diagnosis. Ultrasound Obstet Gynecol 2012; 40 (3): 310-318

2. Olsen R, Doyle Z, Levy $D$, et al. Anomalous pulmonary venous return. Insights into prenatal detection. J Ultrasound Med 2016; 35 (6): 1193-1206.

3. Ishido H, Masutani S, Hishitani T, et al. 'Post-LA space index' as a potential novel marker for the prenatal diagnosis of isolated total anomalous pulmonary venous connection. Ultrasound Obstet Gynecol 2015; 46 (6): 747-748. 